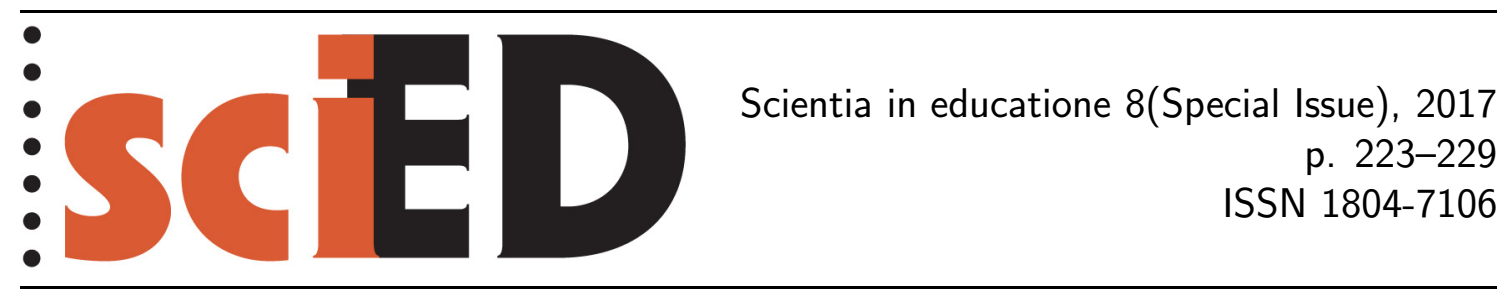

\title{
Using Online Interactive Physics-based Video Analysis Exercises to Enhance Learning
}

\author{
Priscilla W. Laws, Robert B. Teese, David P. Jackson, \\ Maxine C. Willis, Kathy Koenig
}

\begin{abstract}
As part of our new digital video age, physics students throughout the world can use smart phones, video cameras, computers and tablets to produce and analyze videos of physical phenomena using analysis software such as Logger Pro, Tracker or Coach. For several years, LivePhoto Physics Group members have created short videos of physical phenomena. They have also developed curricular materials that enable students to make predictions and use video analysis software to verify them.

In this paper a new LivePhoto Physics project that involves the creation and testing of a series of Interactive Video Vignettes (IVVs) will be described. IVVs are short webbased assignments that take less than ten minutes to complete. Each vignette is designed to present a video of a phenomenon, ask for a student's prediction about it, and then conduct on-line video observations or analyses that allow the user to compare findings with his or her initial prediction. The Vignettes are designed for web delivery as ungraded exercises to supplement textbook reading, or to serve as pre-lecture or pre-laboratory activities that span a number of topics normally introduced in introductory physics courses. A sample Vignette on the topic of Newton's Third Law will be described, and the outcomes of preliminary research on the impact of Vignettes on student motivation, learning and attitudes will be summarized.
\end{abstract}

Key words: video analysis, interactive curricular materials, web-based assignments. 


\section{INTRODUCTION}

Video expositions are already available to help students solve problems, listen to lectures, view demonstrations, and perform virtual laboratory experiments. Although video analysis is becoming popular, materials that combine short video expositions with data collection and the analysis of real phenomena are not yet widely available. The Interactive Video Vignette project (a.k.a. IVV project) involves the creation of a new genre of educational materials. Information on how each student interacts with a Vignette can be tracked automatically, so PIs are acquiring a large body of data on how students interact with a Vignette with regard to: (1) preconceptions; (2) data interpretation abilities; and (3) conclusions. This ongoing research enables the IVV team and others to revise Vignettes to render them then more effective.

As a result of funding from the U.S. National Science Foundation [1] The LivePhoto Physics Group (Muller, 2008) is working on the creation and testing of about 25 short single-topic video expositions. A typical Vignette, designed to take students less than ten minutes to complete, starts by asking students to observe a video of a phenomenon and formulate preliminary predictions about it. After observing to phenomenon more carefully, sometimes in slow motion, or using video analysis to make associated measurements, students are invited to draw conclusions. A physics instructor then summarizes the outcomes of the experiment and briefly discusses how the experimental results exemplify a particular law or phenomenon.

Each vignette is designed for web delivery to supplement textbook reading or serve as a pre-lecture or pre-laboratory activity. These Vignettes are designed to address topics covered in introductory physics courses that can be illuminated with videos and address student learning difficulties identified by Physics Education Research and Cognitive Science (Roth, 1985; [2]). This four-year project began in late 2011. Vignettes that are slated to be available for use by teachers and publishers during 2014 at the comPADRE website [3] are listed in Table 1.

Table 1: Interactive Video Vignettes slated for distribution in 2014

\begin{tabular}{c|c}
\hline Projectile Motion & Newton's First Law \\
\hline Ball Toss Dynamics & Newton's Second Law \\
\hline Slinky Drop Dynamics & Newton's Third Law \\
\hline Ball Drop & Bullet/block experiment \\
\hline
\end{tabular}

\section{A Sample Vignette on Newton's Third LaW}

In order to give readers a better idea of what a Vignette is like, we have chosen to describe our Vignette on Newton's Third Law. This law can be stated quite simply.

Newton's Third Law: If one object is exerting a force on a second object, then the second object is also exerting a force back on the first object. The two forces have exactly the same magnitude but act in opposite directions.

But, we know from the outcomes of physics education research that even when introductory physics students can recite Newton's Third Law, very few of them believe it (Maloney, 1984; Boyle \& Maloney, 1991).

Our Vignette on the Third Law provides a dramatic demonstration of the difficulties students and other people have in understanding this simply stated Law. The Vignette features a series of "person on the street interviews" which demonstrate 
that most people do not believe Newton's $3^{\text {rd }}$ Law, whether or not they have taken introductory physics.

The Vignette starts by asking several interviewees independently what the interaction forces would be like if two identical carts move toward each other at the same speed and collide. Every person who was interviewed said the forces would be equal and opposite. Our "professor" who interviewed people individually did so by showing a video of carts outfitted with forces probes colliding. This video display allowed each person who was interviewed to confirm whether or not he or she was correct.

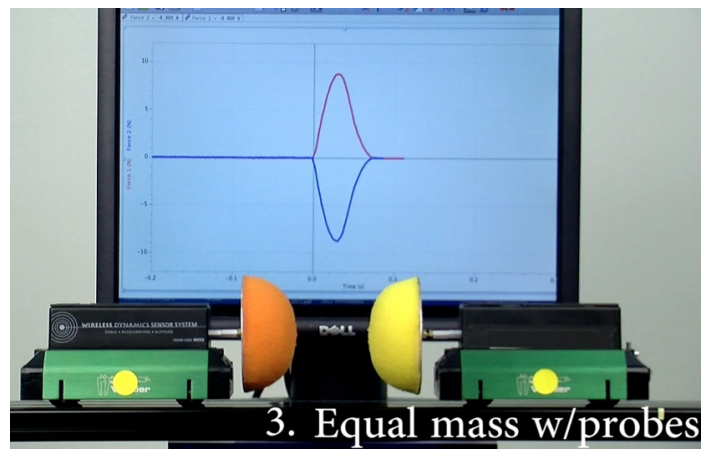

Figure 1: Two identical carts move toward each other at the same speed, collide and then recoil. Force probes readings show that the interaction forces have equal magnitudes on a moment-by-moment basis

In order to consider a more complicated situation that tests people's belief in the Third Law, the professor showed interviewees a video of a real head on car crash in which a larger, faster car collides with a smaller slower car.

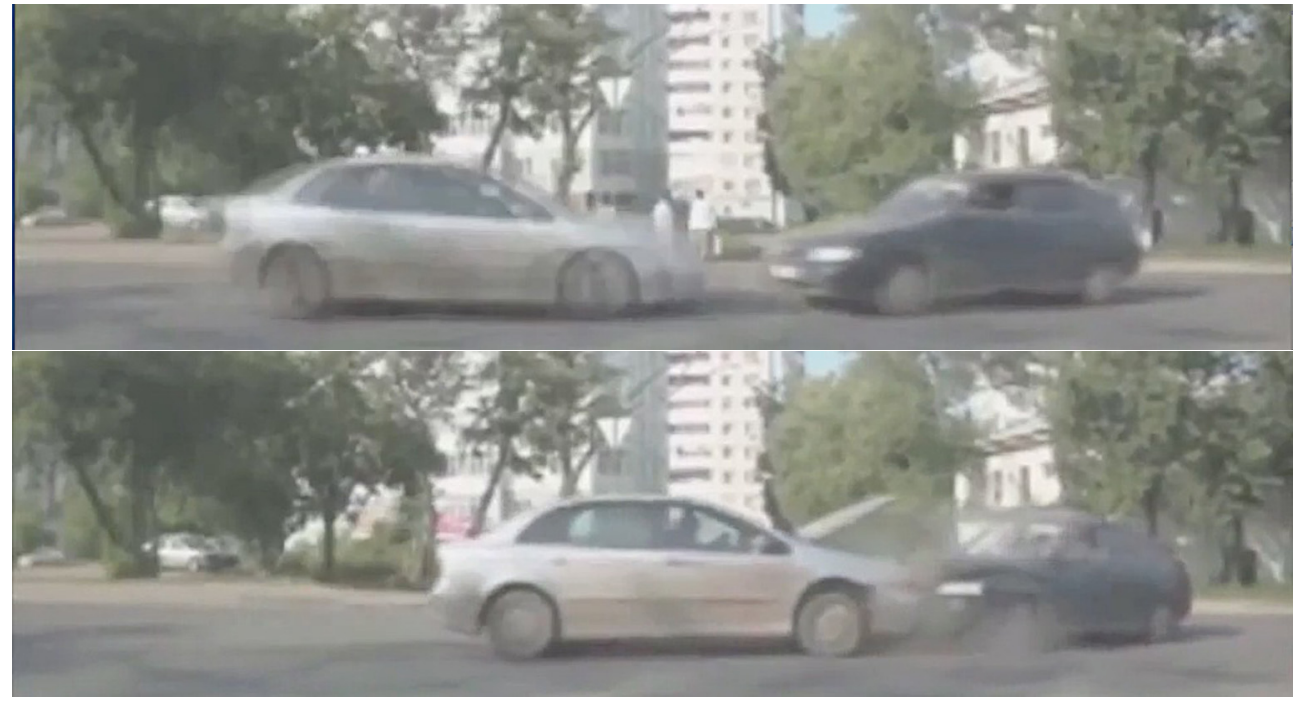

Figure 2: Video frames of two cars undergoing a head on collision

When these interviewees are asked to predict whether there were differences in the interaction forces, if the car on the left has more mass and is moving faster. The IVV team found that ten out of eleven people, who were asked if the forces were different, predicted that the faster more massive car exerts more force on the slower less massive car. The only interviewee who made the "correct prediction" turned out to be a recent secondary school graduate who had just passed an advanced placement examination in physics - not a typical "person on the street" and was, most probably, an above average physics student. 
Since there were no force sensors on the real cars, we showed our interviewees a collision between a cart with extra mass loaded on it and a slower, less massive cart. These interviewees were asked to predict the relative size of interaction forces when one object has more mass than the other and is moving faster. Ten out of eleven of them predicted that the faster more massive cart exerts more force on the slower less massive cart. Next we proceeded to show each interviewee a video of a more massive faster lab cart outfitted with a force sensor exerting an equal and opposite force on a slower, less massive cart that was also outfitted with a force sensor. This result is illustrated in Figure 3.
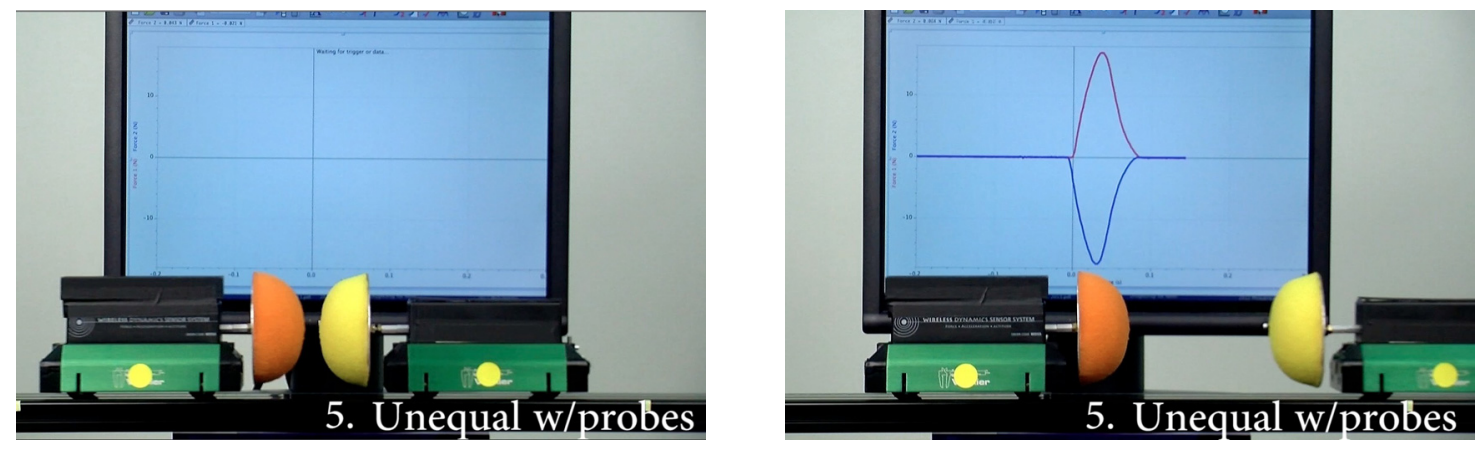

Figure 3: The two video frames show cars of unequal mass just before and after a head on collision. Before the collision the carts move toward each other at the same speed.

After the collision the more massive cart on the left slows down while less massive cart on the left recoils to the right very rapidly

Since the lighter cart recoiled rapidly, it is obvious that in a real situation the driver in the lighter car would feel much more impact. If the passengers are not wearing seat belts, we are also able to demonstrate that the passenger in the slower moving and lighter cart will suffer more damage. This is in spite of the fact that Newton's Third Law still holds for the contact forces between the fronts of the carts!! However, our interviewees intuition is correct - the driver in the smaller, slower cart will indeed be at more risk for injury even though the interaction forces between the two colliding carts are the same! This demonstrates that common beliefs about forces in this situation are generally wrong. However, the driver in the smaller car is still at a higher risk for injury! This is shown in Figure 4 that displays the more massive cart on the left hitting the less massive cart on the right. The rapid recoil of the right cart jolts its driver who falls forward.
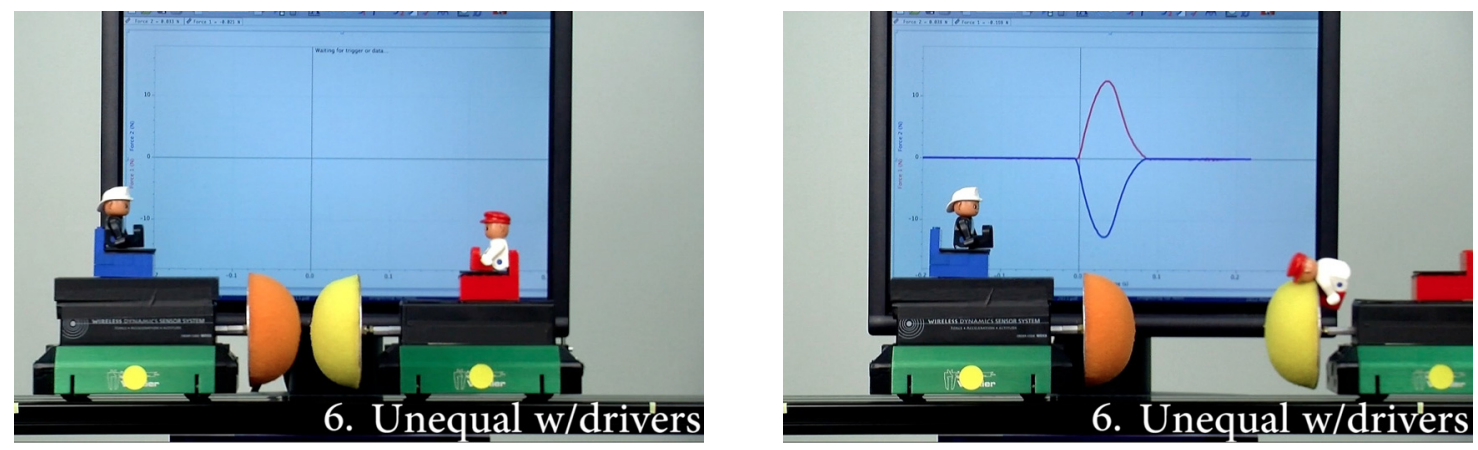

Figure 4: Video frames showing the unequal mass cars and their "drivers" just before and after a head on collision. The driver in the less massive cart shown of the right falls over while the other driver on the left merely slides forward a bit 


\section{PreliminaRy RESEARCH ON IVV USE}

Since Interactive Video Vignettes are being designed to address scientific phenomena and principles that many students tend to misunderstand, the LivePhoto Group recommends that instructors arrange to give their students credit for completing a Vignette. But, our group did not feel that student predictions and other answers to questions should be graded. This led the group to conduct research on how to motivate students to do a vignette that is "assigned by an instructor".

Although the Rochester Institute of Technology and Dickinson College students tested some of the Vignettes, most of the early motivation research was done at the University of Cincinnati in the Winter and Spring Quarters of 2011 and 2012.

1. In the winter quarter 610 students taking one of the sections of calculus-based introductory physics received an email suggesting that they view IVV on Projectile Motion as an optional homework assignment to "help them understand the topic better". Only $28 \%$ of students completed the "suggested" IVV.

2. In the spring quarter 127 students in one of the sections of calculus-based introductory physics received an email suggesting that viewing the IVV on Projectile Motion IVV as an optional homework assignment would help them understand the topic better AND that there would be a related exam question. This time $39 \%$ of the students completed the IVV.

3. In another section at the University of Cincinnati the final exam included a question on the nature of the vertical component of motion associated with the trajectory of a projectile. It turned out that $92 \%$ of the students who had completed the projectile motion IVV answered an exam question about the vertical component of the projectile's motion correctly. On the other hand, only $71 \%$ of the students who didn't complete the related IVV answered the vertical motion question correctly.

A new project involving the impact of student use of IVVs on Projectile Motion and Newton's three laws of motion is underway in introductory classes at University of Cincinnati, Rochester Institute of Technology and Dickinson College. In this study students are being given homework credit for completing each of the four vignettes but not graded on their answers. A pre- and post- test is being administered to the participating students at all three institutions with questions of each of the four topics.

Students who complete IVVs seem to enjoy them. Some of the optional comments collected from students as part of completing their IVV assignments during the Spring of 2012 at the University of Cincinnati include:

"There should be more videos like this to understand concepts."

"Worked Great! Informative and easy to understand!"

"Great! Good way to show proof of concept, I would like for every chapter to have one of these."

"It would be good if there was closed captioning on the video for the hearing impaired."

"GREAT VIDEO!!! WOOOHOOO PHYSICS!!!"

"I thought the interactive video was very well made. I can't wait to see and learn more." 


\section{CONCLUSIONS}

Members of the LivePhoto Physics Group who have participated in the design and testing of the Interactive Video Vignette Project remain enthusiastic about the potential of Video Vignettes as a viable alternative to on-line lectures and other on-line teaching modalities that are primarily passive. In addition, our group is optimistic that ongoing research on the effectiveness of IVVs on other topics will prove to be superior to many conventional out-of-class assignments and in some cases augment or replace other types of out-of-class learning experiences.

Readers who would like to try NSF supported IVVs can access the collection on the comPADRE website (http://www.compadre.org/ivv/). Currently the authors are working with Cengage to create an extended series of IVVs to be widely disseminated in the future.

\section{REFERENCES}

Boyle, R. K. \& Maloney, D. P. (1991). Effect of written text on usage of Newton's third law. J. Res. Sci. Teach., 28(2), 123-140. [5]

Maloney, D. P. (1984). Rule-governed approaches to physics: Newton's third law. Phys. Educ., 19(1), 37-42. [4]

Muller, D. A. (2008). Designing effective multimedia for physics education [Dissertation thesis]. U of Sydney.

Roth, K. J. (1985). Conceptual Change Learning and Students' Processing of Science Texts. In Annual Meeting of the American Educational Research Association.

\section{Notes}

[1] Supported by NSF grants DUE-1122828 and DUE-1123118.

[2] The LivePhoto Physics group includes Priscilla Laws, David Jackson \& Maxine Willis (Dickinson College), Robert Teese (Rochester Institute of Technology), Patrick Cooney (Millersville University), and Kathy Koenig (University of Cincinnati). A collection of educational videos is available on the group's website http://livephoto.rit.edu/

[3] As materials become available they can be accessed at http://www.compadre.org/

[4] More than 100 university students with different background in physics were asked to compare the forces that two interacting objects exerted on each other. About $2 / 3$ of the students thought that they would be of different magnitude in some circumstances.

[5] The investigators examined the beliefs about Newton's third law of 100 university students before instruction. Half of the students were given a handout describing forces with explicit statements of the third law. No student without the handout applied the third law correctly and of those with the handout, fewer than half applied it correctly. 
PRISCILLA W. LAWS

DAVID P. JACKSON

Maxine C. Willis

Dickinson College, Carlisle PA, United States

ROBERT B. TEESE

Rochester Institute of Technology, Rochester, NY, United States

KATHY KoEnIG

University of Cincinnati, Cincinnati, OH, United States 